\title{
Implementation of Merchant CRM system based on MVC architecture
}

\author{
Vignesh Ganesh $^{1}$, Nikita Singh ${ }^{2}$, Thaksen J. Parvat ${ }^{3}$ \\ Student, Department of CSE, Sinhgad Institute of Technology, Lonavala, India ${ }^{1,2}$ \\ Associate Professor, Department of CSE, Sinhgad Institute of Technology, Lonavala, India ${ }^{3}$
}

\begin{abstract}
CRM (Customer Relationship Management) is a business process in which client relationships, customer loyalty and brand value are built through marketing strategies and activities. A payment gateway is a server that is dedicated to linking websites and banks so that online credit card transactions can be completed in real-time. This paper attempts to introduce that:- There are hundreds of different CRM systems existing in the market but based on MVC pattern, the CRM system model can increase performance, provide high reliability, increase in speed and save time. Implementation of CRM designed solely for the merchant so as to ease his transactions which will be based on the MVC architecture will be highly productive.
\end{abstract}

Keywords: Merchant, CRM, MVC, Customer, Payment

\section{INTRODUCTION}

CRM as a service means understanding customer needs if today is the user's birthday, or the totals, taxes, and and involves tracking of information on products and shipping charges for a shopping cart). Most applications services with a view to understand the thinking of the use a persistent storage mechanism such as a database to customer and to develop a stronger relationship.

A CRM for merchant involves understanding the needs and market trends for a merchant. It also allows merchant to sell their products to all over the world and track their transactions through one click. The world population is using online payments. There is a need of a secure architecture and flow for a merchant so as to avoid his losses. Many Merchants provide the ease of shopping to the customers all over the world siting at their home using E-commerce websites. Most of the population is using online payments hence there is need of a secure online payment gateway.

Also with the increasing demands for the online shopping the overhead on the Merchant's side has also increased as it has to maintain its customers details, online transactions made whether successful, unsuccessful or pending along with finding new methods for increasing its customer relationships. So there is a need for a stable, secure, reliable, high performance Merchant CRM system model. All of these characteristics can be achieved using a MVC framework.

\section{OVERVIEW OF MVC}

MVC, abbreviation for "Model-View-Controller" is the name of a methodology or design pattern for successfully and efficiently relating the user interface to underlying data models. The MVC helps to manage complex applications, as the user can focus on one aspect at a time. The model-view-controller pattern proposes three main components or objects to be used in software development:

- The Model is the domain-specific representation of the information on which the application operates. Domain logic adds 'meaning' to raw data (e.g., calculating store data. MVC does not specifically mention the data access layer because it is understood to be underneath, or encapsulated by, the Model.

- $\quad$ The View renders the model into a form suitable for interactions, typically a user interface. Multiple views can exist for a single model, for different purposes. In a Web application the view is usually rendered in a 'Web format' like HTML, XML or JSON. However there are some cases where the view can be expressed in a binary form, e.g. dynamically rendered chart diagrams.

- $\quad$ The Controller responds to events (typically user actions) and processes them, and may also invoke changes on the model. In a Web application, events are typically HTTP requests: a Controller listens for HTTP requests, extracts relevant data from the 'event', such as query string parameters, request headers... And applies changes on the underlying model objects.

The MVC pattern works in four steps. Firstly, the user sends the HTTP request through the User Interface which is a browser to the controller after analysing the request, the controller sends it to appropriate model.

Secondly, the model uses suitable functions and mechanisms handling with the logic of the task according to the request, and returns the data to the controller.

Thirdly, the controller calls correct view, sending the results to the browser which is dealt by the model. Finally, the controller send the HTTP response to the user. 


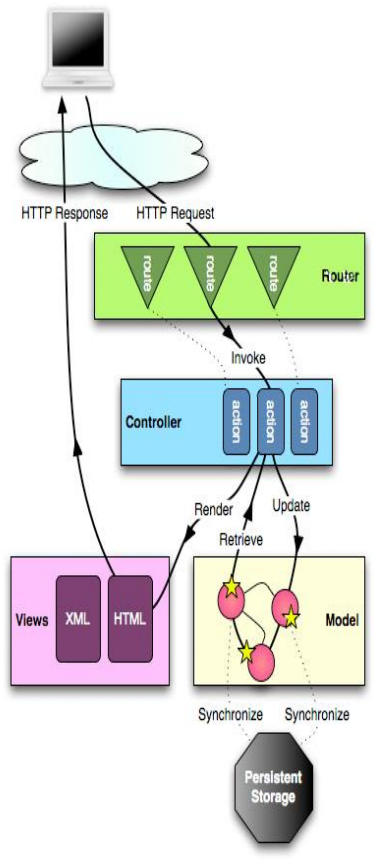

Fig. 1. Working of MVC architecture

\section{III.IMPLEMENTATION OF MERCHANT CRM BASED ON MVC}

As the growth of business in market is increasing, the merchants needs a stable, reliable and efficient CRM system to manage its clients in order to stimulate their future business. Thus, the CRM with MVC is a good solution. MVC keeps the three logic types independency to build the business and data logic oriented business and design the control and display logic oriented application. The changing of business processing does not modify the business and data logic. The changing of business principles and algorithms only modifies Model class. So MVC separates the data accessing and display and ensure the modules independency.

The CRM system based on MVC framework uses "B/S architecture" where "B/S" stands for "Browser/Servers". It is easy to maintain and update the system since it only has to do with the servers. It also suffices the expansibility of user's needs.

The whole system can be divided into several parts: system environment management, user and access control management, fundamental data management, business data management. Each parts function module serves as the business logic layer in MVC, present in different operation based on different information input. Among all the modules mentioned above, the access control module is the key of the system security.

The development platform should be open-source software and with advantages such as less storage room, cross platforms, high performance, low cost, secure and stable.

\section{IV.PRELIMINARIES REQUIRED FOR MERCHANT CRM}

There are three main requirements for a merchant CRM to work:

1) A website that meets the security and eligibility requirements for the bank to issue a merchant account

2) A merchant account from a bank. A merchant account is a special kind of bank account that can be linked directly to a payment gateway. These bank accounts are issued only upon review of a website to ensure it meets privacy and security requirements. Requirements often include an SSL certificate and a privacy policy.

3) A payment gateway provider. Payment gateway providers offer the server that acts as the link between your website and the bank. The server has a very special configuration and a very high level of security due to the sensitive nature of the data being transferred.

Once all three requirements are met, the merchant CRM can be designed on the MVC framework. This will then allow for real-time credit card processing.

\section{IMPLEMENTATION OF CRM WITH MVC}

Merchant CRM system gets divided into the three components. View acts as the main GUI where the merchant enters his details access the main functions for the transactions. Controller is the main section where the merchant handles the database and transactions required and which are currently under processing. Model handles all the synchronising of the view and controller section. Once this The main advantage of integrating the CRM system with payment gateway is that the merchant is free from issues of setting up SSL at their website and worrying about the privacy of the customer's credit card details.

\section{CONCLUSION}

The MVC is a better design for CRM system model based on MVC framework as the extension and alternation of CRM system functions only reorganize the corresponding business logic without modifying the whole system. The detachment of the Model layer and View layer makes it easier to change the business logic and reduces the maintenance cost. So the system design based on MVC fits the multi-user CRM system that is extensible, high interactive, and easy to maintain.

\section{REFERENCES}

[1] Integration of CRM Systems with Payment Gateway. International Journal of IT, Engineering and Applied Sciences Research (IJIEASR), 2014

[2] Application of MVC Platform in Bank E-CRM. International Journal of u-and e-Service, Science and Technology, 2013.

[3] https://www.playframework.com/documentation/1.0/main from the website

[4] Application of MVC Platform in Bank E-CRM. International Journal of u-and e-Service, Science and Technology, 2013.

[5] Nature-Inspired Techniques in the Context of Fr aud Detection Mohammad Behdad, Luigi Barone, Mohammed Bennamoun, and Tim French1094-6977/\$31.00 @ 2012 IEEE 